\title{
Importancia de la citología exfoliativa en el diagnóstico de lesiones bucales
}

Importance exfoliative cytology in the diagnosis of oral lesions
Importância da citologia esfoliativa no

diagnóstico de lesões orais
Fecha de Recepción

19 de junio de 2014
Aceptado para su publicación

10 de septiembre de 2014

\section{María Silvia Almirón}

Profesora Adjunta. Cátedra de Anatomía Patológica. Facultad de Odontología. U.N.N.E.

E-mail: msalmiron@odn.unne.edu.ar

María de las A. Montenegro Profesora Adjunta. Cátedra de Patología General y Sistemática. Facultad de Veterinaria. U.N.N.E. E-mail:patgral@vet.unne.edu.ar

\section{María Alejandra Gili}

Profesora Adjunta. Cátedra Histología y Embriología. Facultad de Odontología. U.N.N.E.

E-mail: magili@odn.unne.edu.ar

\section{Silvia Mariana Segovia} Auxiliar Docente de Primera Categoría. Cátedra Histología y Embriología. Facultad de Odontología. U.N.N.E. E-mail:smsegovia@odn.unne.edu.ar

\section{Resumen}

Toda patología comienza a nivel celular presentando cambios morfológicos o funcionales, por lo que es de suma importancia el empleo de técnicas que permitan la observación de dichos cambios y lleven a un diagnóstico certero y por ende a un adecuado tratamiento'.

La citología exfoliativa es un procedimiento simple e incruento que ha sido objeto de controversia sobre su validez real en patología oral. En los últimos tiempos ha resurgido en base a su aplicación en el precáncer y el cáncer oral, tanto como metodología diagnóstica como predictiva y para la monitorización de los pacientes ${ }^{2}$.

Actualmente el diagnóstico a través de la citología se ha traducido en el desarrollo de nuevas técnicas como las preparaciones de base líquida, el uso de esta técnica es considerada como una herramienta auxiliar para el diagnóstico de lesiones de la mucosa bucal y actualmente ha despertado un renovado interés por su aplicación en técnicas de biología molecular.

\section{Palabras claves}

Citología. Exfoliativa. Base liquida. Lesiones bucales. Diagnóstico.

\section{Abstract}

All disease begins at the cellular level by presenting morphological or functional changes, so it is very important the use of techniques for 
REVISTA FACULTAD DE ODONTOLOGÍA

ISSN No 1668-7280 - Vol. VIII № 1 - 2015

27
DIVULGACION

Importancia de la citología exfoliativa en el

diagnóstico de lesiones bucales observing these changes and lead to an accurate diagnosis and therefore to an appropriate treatment'.

Exfoliative cytology is a simple and harmless procedure, which has been the subject of controversy over its real validity in oral pathology. In recent times it has resurfaced on the basis of its application in oral precancer and cancer, as well as predictive and diagnostic methodology for monitoring patients ${ }^{2}$.

Currently the diagnosis by cytology has resulted in the development of new techniques such as liquid-based preparations, use of this technique is considered as an auxiliary tool for the diagnosis of lesions of the oral mucosa and currently has sparked renewed interest in its application in molecular biology techniques.

\section{Keywords}

Cytology. Exfoliative. Liquid foundation. Oral lesions. Diagnosis.

\section{Resumo}

Toda a doença começa no nível celular, apresentando alterações morfológicas e funcionais, por isso, é muito importante o uso de técnicas de observação destas alterações e conduzir a um diagnóstico preciso e, por conseguinte, a um tratamento adequado'.

Citologia esfoliativa é um procedimento simples e inofensivo, que tem sido objecto de controvérsia sobre a sua real validade em patologia oral. Nos últimos tempos ele ressurgiu com base na sua aplicação em pré-câncer bucal e câncer, bem como métodos de previsão e de diagnóstico para o acompanhamento de pacientes ${ }^{2}$.

Actualmente o diagnóstico por citologia resultou no desenvolvimento de novas técnicas, tais como preparações à base de líquidos, a utilização desta técnica é considerada como uma ferramenta auxiliar para o diagnóstico de lesões da mucosa oral e atualmente provocou renovada interesse em sua aplicação em técnicas de biologia molecular.

\section{Palavras chave}

Citología. Esfoliativa. Base líquida. As lesões orais. Diagnóstico.

\section{Introducción}

La citología exfoliativa es un proceso simple y no invasivo, este método se utiliza para el estudio de células que se exfolian de los tejidos, las cuales se analizan por medio de coloraciones convencionales ${ }^{3}$. Este examen, conocido como frotis citológico convencional, fue originalmente ideado para la detección precoz de células cervicales cancerosas ${ }^{4}$. La citología exfoliativa se define como el estudio morfológico basado en los caracteres microscópicos de células y componentes extracelulares desprendidos de los órganos espontánea o artificialmente, a través de procedimientos, que en general, son de fácil procesamiento, menos invasivos y más económicos que la biopsia ${ }^{5}$.

La citología se viene aplicando al diagnóstico de las enfermedades orales desde que Papanicolaou y Traut demostraran su validez para el diagnóstico de las neoplasias del cérvix uterino. Presenta una sensibilidad del 52 al 64\% presentando el inconveniente de los falsos negativos que genera y los falsos positivos debido a la toma en lugares donde se desarrolle queratina, exista sepsis, necrosis o sangrado6.

Actualmente con el progreso de la técnica citológica, que se ha traducido en el desarrollo de preparaciones de base líquida, el uso de esta técnica como herramienta auxiliar en el diagnóstico de lesiones de la mucosa oral ha despertado un renovado interés. En esta técnica se introduce el instrumento de obtención de la muestra en su totalidad en un medio líquido, fijando las células y evitando la degeneración por el aire ${ }^{7}$.

En las preparaciones de base líquida, la muestra y el dispositivo de recolección se transportan en un recipiente que contiene un líquido conservador que permite la inmediata fijación de las células. Como todos los métodos de citología de base líquida permiten la obtención de más de una preparación por cada muestra recogida, pudiendo utilizarse otras coloraciones como PAS y Plata Metamina, además de la tinción de Papanicolaou. La citología a base líquida presenta la ventaja de larga vida útil y, por lo tanto, está disponible para los exámenes adicionales que sean necesarios.

Los sistemas de procesamiento más utilizados son la sedimentación y la centrifugación. Mediante el procesado de las muestras con esta técnica, 
se soluciona el problema de la mala calidad de las muestras debido a su grosor, permitiendo así un extendido uniforme de la misma en monocapa. Además es posible la conservación del material recolectado para realizar estudios simultáneos o posteriores de biología molecular 8 .

Esa técnica permite obtener preparaciones con abundancia de células dispersas en una capa fina y homogénea, sangre, inflamación y mucus quedan reducidos y distribuidos por toda la preparación. El fondo claro que así se obtiene aumenta la sensibilidad y la calidad de la muestra. Comparado con los frotis convencionales, el uso de preparaciones de base líquida ha permitido reducir considerablemente el número de preparaciones insatisfactorias o satisfactorias pero limitadas, debido a las características del ejemplar, lo que disminuye el número de resultados falsos negativos ${ }^{4}$.

Aunque la citología convencional es todavía el principal método usado para los exámenes rutinarios de frotis, este estudio demostró que, entre otras ventajas, la citología de base líquida logra una mejor calidad en la resolución de la morfología celular. Su desventaja reside en que exige tanto un laboratorio con equipo más sofisticado como personal mejor entrenado para manejar debidamente el proceso y analizar las muestras.

La citología exfoliativa oral se define como el estudio e interpretación de los caracteres de las células que se descaman, natural o artificialmente, de la mucosa oral. Están indicadas en lesiones erosivas, ulceradas o rojas; enfermedades vesículo- ampollares (pénfigo, virus herpes y varicela zoster), micosis (candidiasis y otras micosis profundas), cáncer bucal, detección de

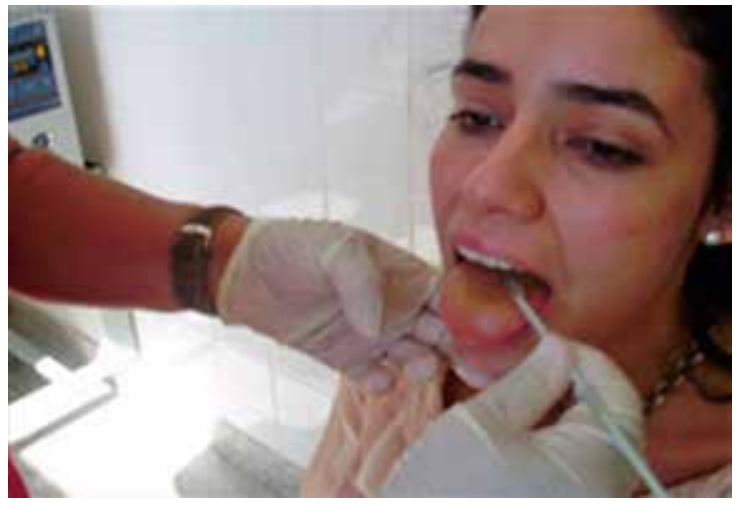

Figura I. Toma citológica. células anaplásicas. Las células orales que se van a analizar tras su extendido, pueden obtenerse mediante diferentes sistemas físicos de raspado de la superficie mucosa, o mediante enjuagues de la cavidad oral o bien mediante una toma de muestra de saliva de los pacientes.

También en individuos fumadores con mucosa oral clínicamente sana, se han observado cambios citológicos como una mayor queratinización, existiendo también reportes de un mayor grado de actividad nucleolar. En estos estudios, las células para frotis se han obtenido por medio de espátula de madera?.

Es una técnica sencilla, no agresiva, indolora y aceptada por los pacientes ${ }^{10}$, por lo que podría ser útil en el diagnóstico precoz del cáncer oral. Consiste en observar al microscopio la morfología de las células epiteliales superficiales después de su toma, fijación y tinción. La misma revela cambios morfo-fisiológicos en las células epiteliales, tales como cambios en el tamaño nuclear y aspecto sucio del preparado, los cuales ayudan a un diagnóstico presuntivo, su valor principal consiste en que sin destruir la integridad del tejido, posibilita la extracción repetida de células superficiales de la encía y la mucosa bucal con fines diagnósticos.

La técnica con la que se obtiene un mejor material es la de raspado, que se realiza a expensas de la separación mecánica del epitelio mucoso con diferentes instrumentos ${ }^{4}$. El instrumento empleado para realizar la toma citológica debe ser fácil de usar en cualquier localización, provocar pocas molestias y proporcionar un número adecuado de células epiteliales.

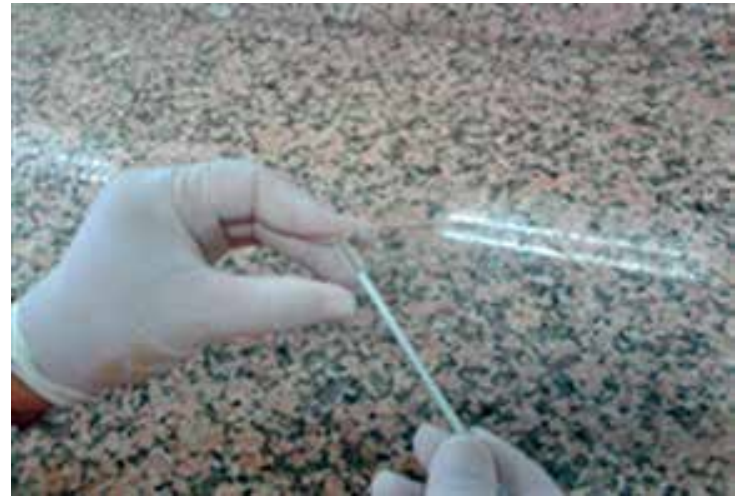

Figura 2. Extendido citológico. 


\section{Instrumentos para toma citológica}

El cepillo o cytobruh es un elemento adecuado, debido a la facilidad en la toma y a la calidad de la muestra citológica bucal este parece aumentar el número de células recogidas por muestra, y permite una mejor distribución de las mismas en el porta-objetos, lo que podría aumentar la sensibilidad de la técnica".

El cáncer bucal es la neoplasia maligna más frecuente en la región de cabeza y cuello, siendo el carcinoma de células escamosas el que presenta mayor incidencia. La mayoría de las lesiones bucales son benignas, pero muchas tienen características que la confunden fácilmente con una lesión maligna. Una parte significativa de estos cánceres se desarrolla a partir de desórdenes bucales potencialmente malignos como la leucoplasia.

Hay conclusiones que señalan que las principales ventajas clínicas de la citología exfoliativa se deben a que es una técnica rápida, lo que permite que sea practicada en lesiones sospechosas ya identificadas o en el seguimiento de lesiones malignas post tratamiento ${ }^{12}$.

El cáncer bucal puede debutar como una pequeña mancha blanquecina o eritematosa, una úlcera plana, en forma exofítica, excavada, infiltrante o nodular ${ }^{13}$.

La leucoplasia es la lesión precancerosa más común de la mucosa oral, siendo variados los factores que promueven su desarrollo ${ }^{14}$.

La transformación maligna en la cavidad oral depende en cierto modo del sitio anatómico que se ve afectado (labios, lengua, piso de la boca, paladar, gingiva, mucosa bucal y orofaringe), de

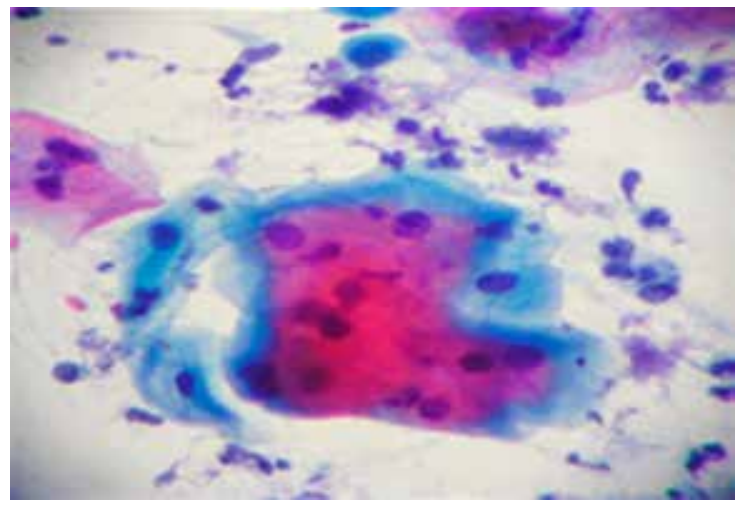

Figura 3. PAP. Células epiteliales superficiales. los numerosos factores endógenos y exógenos que afectan el crecimiento de las células malignas en cada uno de estos sitios y de la relación con el suministro de nutrientes ${ }^{15}$.

Actualmente la vía más efectiva para disminuir la morbimortalidad del cáncer bucal es el diagnóstico precoz seguido por un tratamiento adecuado .La aplicación de la citología en la detección de los fenómenos displásicos ha sido motivo de múltiples estudios con diferentes resultados.

Una de las principales causas del fracaso en el tratamiento del carcinoma de células escamosas es que una proporción significativa de estos casos no son diagnosticados o tratados hasta que alcanzan un estado avanzado de desarrollo ${ }^{7}$. Esto se debe a que los pacientes habitualmente no reportan características inusuales en su boca y muchos profesionales de la salud no investigan a fondo las lesiones observables. La detección precoz en estadios asintomáticos garantiza no sólo un aumento en las tasas de supervivencia, sino también una mejor calidad de vida, como consecuencia de tratamientos menos agresivos y mutilantes.

Los métodos diagnósticos clásicos para las lesiones cancerosas y precancerosas orales son el examen clínico y el estudio histopatológico del material obtenido por biopsia. El análisis biópsico es todavía la técnica más aceptada para determinar de un modo fiable la naturaleza de las lesiones de la mucosa oral ${ }^{4}$.

En gran medida el reciente interés por el estudio de la citología oral en la patología cancerosa oral, se ha debido a la aplicación de nuevas técnicas moleculares. El estudio genético de

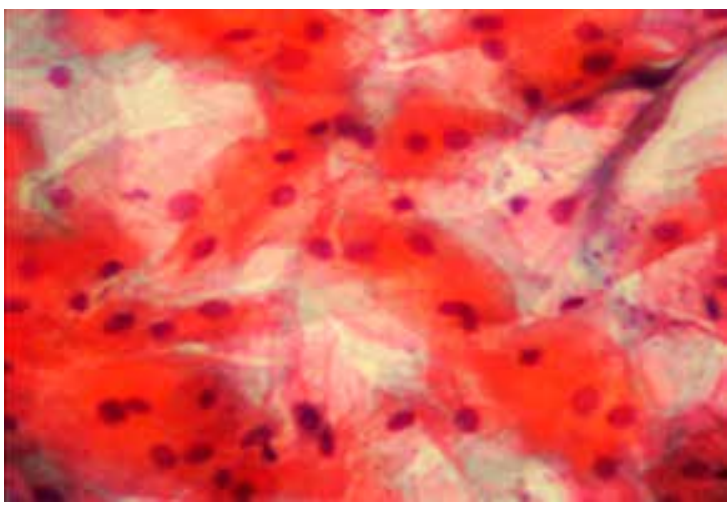

Figura 4. PAP. Cél. Sup. queratósicas. 


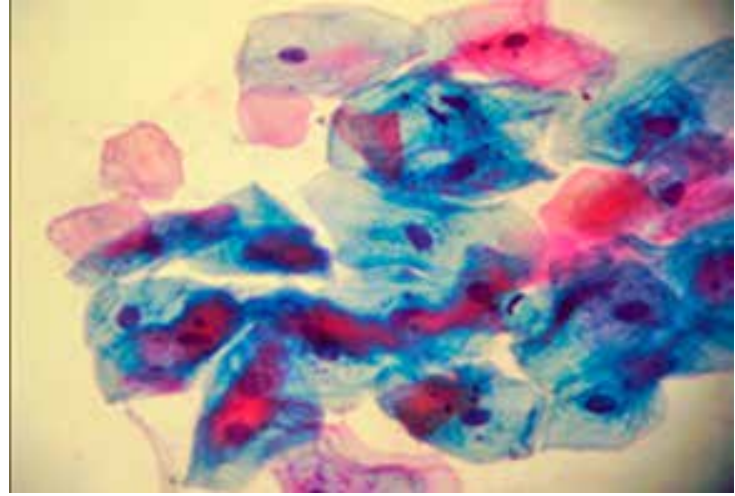

Fig.5. Células epiteliales sup. Anucleadas

marcadores moleculares permite detectar alteraciones antes de que se produzcan cambios en la morfología celular y de que esos cambios sean clínicamente visibles. Mientras que la evaluación citológica oral clásica es una labor intensa que requiere un elevado grado de experiencia para la identificación y valoración de células con morfología sospechosa, el análisis de las alteraciones moleculares es objetivo, y trata de identificar anomalías génicas específicas ${ }^{16}$.

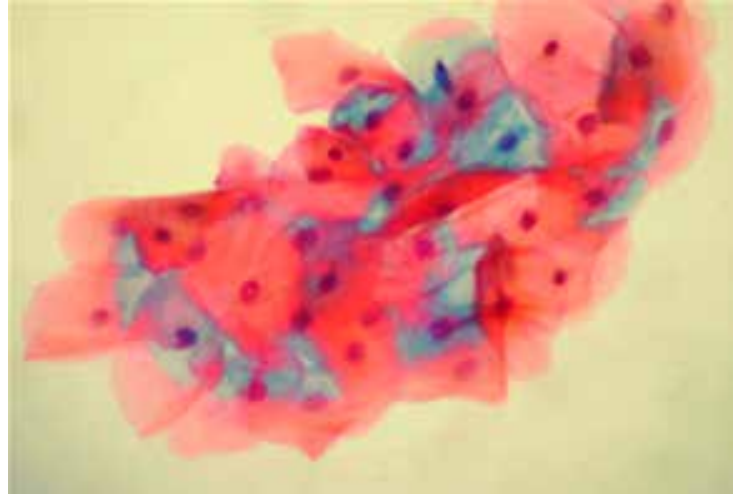

Fig.6 Cel.epiteliales superf. e intermedias.

\section{Conclusión}

La citología exfoliativa, constituye un método seguro, simple y rápido, que no tiene contraindicaciones para efectuarlo. Presenta un alto porcentaje de sensibilidad, alto valor predictivo positivo y negativo, una coincidencia diagnóstica elevada y un gran porcentaje de eficiencia global, lo que demuestra su importancia como método no invasivo de diagnóstico

Se destaca también la significación de la habilidad y experiencia del citólogo en la certeza diagnóstica del método para una correcta interpretación de las alteraciones celulares observadas.

La utilización de la citología exfoliativa vuelve adquirir importancia como técnica diagnóstica para obtener muestras para la aplicación de técnicas sofisticadas de diagnóstico, citomorfometría, análisis del contenido de ADN, y análisis molecular, ganando un espacio como método de diagnóstico fiable del cáncer oral en sus estadios más precoces. 
REVISTA FACULTAD DE ODONTOLOGÍA

ISSN No 1668-7280 - Vol. VIII № 1 - 2015

31
DIVULGACION

Importancia de la citología exfoliativa en el

diagnóstico de lesiones bucales

\section{Bibliografia}

I. Jerez, Elsy. Zerpa, Reina. Omaña, Carlos. Determinar el uso de la citología oral como medio de diagnóstico en la facultad de odontología de la Universidad de los Andes Merida Venezuela. Acta Bioclinica. Volumen 3, $\mathrm{N}^{\circ}$, enero-junio 2013.

2. Acha Amelia, Ruesga María T., Rodríguez María J., Martínez de Pancorbo María A., Aguirre José M. Aplicaciones de la citología oral por raspado (exfoliativa) en el cáncer y precáncer oral. Med. oral patol. oral cir. bucal (Ed. impr.) [revista en la Internet]. $2005 \mathrm{Abr}$ [citado 2015Sep 14] ;I0(2): 95-102. http://scielo.isciii. es/scielo.php?script=sci_arttext\&pid=S16984447200500020000I\&Ing=es.

3. Vargas Domínguez, Reynaldo. Manrique Camacho, María Jovita. Citología Exfoliativa y Punción con aguja fina. Una herramienta efectiva en el consultorio dental. Odontología Actual / año 5, núm. 58, febrero de 2008.

4. Ceccotti-Sforza. El diagnóstico en clínica Estomatológica. Editorial Médica Panamericana. S. A. 2007. Pág. 14.

5. Hayama Fábia H, Motta Ana CF, Silva Antonio de Padua G, Migliari Dante A. Preparaciones de base líquida vs. citología convencional: Adecuación de las muestras y coincidencia de diagnóstico en lesiones orales. Med. oral patol. oral cir. bucal (Ed. impr.) [revista en la Internet]. $2005 \mathrm{Abr}$ [citado 2015 Sep 14]; 10(2): II5-122. http://scielo.isciii. es/scielo.php?script=sci_arttext\&pid=S169844472005000200004\&lng=es.

6. Albornoz López del Castillo Carlos, Rivero Pérez Oscar, Bastian Manso Luis. Avances en el diagnóstico de las lesiones cancerizables y malignas del complejo bucal. AMC [revista en la Internet]. 2010 Oct [citado 20I5Sep I4]; I4(5): http://scielo. sld.cu/scielo.php?script=sci_arttext\&pid=SI02502552010000500019\&Ing=es

7. Ricci A Paolo, Perucca P Ernesto, Koljanin V Josip, Baeriswyl T Eduardo. CITOLOGÍA DE BASE LÍQUIDA: REVISIÓN DE LA HISTORIA Y LOS ESTUDIOS AL RESPECTO. Rev. chil. obstet. ginecol. [Internet]. 2004 [citado 2015 Sep 14]; 69( 3 ):256-262. http://www.scielo. $\mathrm{cl} / \mathrm{sciel}$.php?script $=$ sci_arttext\&pid=S07 17 752620040003000 I 4\&Ing=es.

8. Reboiras López, María Dolores. Estudio comparativo de tres instrumentos para la toma de muestras citológicas de la cavidad oral: cytobrush, oral cdx y cureta dermatológica. Santiago de Compostela, Julio del 2010.
9. Orellana Bustos, A. I. Espinoza Santander, I. L. Franco Martínez, M.E. Lobos Jaimes-Freyre, N. Ortega Pinto, A.V. Evaluación del grado de queratinización y el recuento de AgNORs en citología exfoliativa de mucosa oral normal de individuos fumadores y no fumadores. Med Oral 2004; 9:197-203.

10. Rocha Buelvas A. Oral cancer: the role of the dentist in early diagnosis and control. Rev.Fac. Odontol. Univ. Antioq. 2009; 2I(I): II2-12I.

II. Dericia, J. I. Ignacio, Gonzalez Segura-MariaBrezzo, Luis Marcelo Croharé Dante Gustavo, Secchi Alicia Inés, Malberti, Ana María, Zarate. Estudio citomorfométrico de pacientes con cáncer y desórdenes potencialmente malignos de la cavidad bucal. Acta Odontológica Venezolana. Vol. $50 \mathrm{~N}^{\circ} 3 / 2012$.

12. Álvarez N, Rosallyn G. Carrero T, Jenny F. Omaña C,Carlos J. Florido P, Rosalba.Cambios celulares presentes en mucosa palatina conestomatitis subprotésica.Trabajo de investigación. Vol. 7 - N 2 - julio-diciembre -2012.12 - 20.

13. López del Castillo,Carlos Albornoz. Zequeira Peña, Jorge Luis. Merino López, Clemente. Siré Gómez, Alexis. EL DIAGNÓSTICO CLÍNICO Y LA DETECCIÓN PRECOZ DEL CÁNCER BUCAL.Archivo Médico de Camagüey 2003; 7(5).

14. Deyanira Carnesoltas-Lázaro. Aníbal Domínguez-Odio. Ana Iris Frías-Vázquez, Carlos Manuel Dutok-Sánchez. Gilda García-Heredia. Alteraciones citogenéticasbucoepitelialesen pacientes portadores de leucoplasia.RevMex Patol Clin, Vol. 54, Núm. 3, pp 104-III • Julio - Septiembre, 2007.

15. Brunotto, Mabel. Zárate, Ana María. Cismondi, Adriana. Fernández, María del Carmen. Noher de Halac, Rita Inés.Valoración de la citología exfoliativa como factor de predicción en lesiones de la mucosa oral. Med Oral Patol Oral Cir Bucal 2005; Suppl 2: E92-102.

16. Diniz Freitas Márcio, García García Abel, Crespo Abelleira Antonio, Martins Carneiro José Luis, Gándara Rey José Manuel. Aplicaciones de la citología exfoliativa en el diagnóstico del cáncer oral. Med. oral patol. oral cir. bucal (Ed.impr.) [revista en la Internet]. 2004 Oct [citado 2015 Sep 14]; 9 (4): 355-36I. Disponible en: http://scielo.isciii. es/scielo.php?script=sci_arttext\&pid=S1698444720040004000 I4\&Ing=es. 